\title{
Acupuncture versus medical treatment for migraine and muscle tension headaches
}

\author{
L LOH, PW NATHAN, GD SCHOTT, KJ ZILKHA \\ From the National Hospital for Nervous Diseases, Queen Square, London, UK
}

SUMMARY In 48 patients with chronic migraine and muscle tension headaches, a comparison was made between a prophylactic course of acupuncture and of medical treatment. It was intended that all patients should have 3 months with both forms of treatment, but 19 were unwilling to change from one form of prophylaxis to the other. Twenty-four of 41 patients improved on acupuncture, the improvement being very marked in nine; nine of 36 patients improved on medical treatment, the improvement being marked in three. Of the 29 patients who changed from one form of treatment to the other, a larger proportion preferred acupuncture to medical treatment. A beneficial response to acupuncture was more likely when the patient had local tender muscular points. The presence of depressive features did not preclude satisfactory treatment with acupuncture. No major side effects were encountered with acupuncture.

The thousands of patients attending general practitioners, hospitals, migraine clinics and non-medical practitioners and the profusion of drug regimes and physical methods offereu, all bear witness to the fact that the treatment of migraine and other sorts of headache is unsatisfactory. That this is so is realised by the public, who have become more interested in treatments that are offered outside the medical profession. Acupuncture in this country is provided by a great many non-medically qualified persons and a few doctors of medicine. Although uncontrolled clinical studies in more than twenty thousand patients at UCLA suggest that it may be a useful form of treatment for migraine and muscle tension headaches, ${ }^{1}$ it continues to be considered a "fringe technique".?

In order to assess the usefulness of acupuncture in migraine and muscle tension headaches, a trial of acupuncture and standard medical treatment was undertaken; the results are presented in this report. The purpose of treatment was to prevent migraine and headaches or to reduce their number and severity; it was not to treat the headache when it occurred.

Address for reprint requests: Dr P Nathan, National Hospital for Nervous Diseases, Queen Square, London WC1N 3BG, UK.

Received 9 August 1983 and in revised form 20 October 1983. Accepted 28 October 1983

\section{Patients and methods}

We did not classify migraine and muscle tension headaches separately, for the following reasons. From using acupuncture before we started this trial, we already knew that acupuncture was an effective prophylactic for both kinds of headaches. Patients commonly have both kinds of headache and they frequently do not differentiate them when they report their progress. Although there are clearcut examples of migraine and of muscle tension headaches, there are many headaches where it is artificial to assign them to one or other of these two categories.

This trial comprised 55 patients referred to a consultant neurologist at The National Hospital for Nervous Diseases, Queen Square, London, who agreed to participate in this project. Almost all the patients had had many months or years of drug treatment before being referred here. It will be realised that the patients sent to this hospital on account of migraine or other kinds of headache are a selected group. They come here because their general practitioners suspect that the diagnosis is not certain, because routine treatment of their headaches has failed, because they have had headaches for a long time, or because they are dissatisfied with the treatment they have had.

Seven of the 55 patients who entered the trial failed to complete it, and so the trial is based on 48 patients. Of these seven patients, three attended too irregularly to assess, two were lost to follow-up as administrative arrangements were unsatisfactory, one had too few headaches to warrant inclusion in the trial and one found acupuncture too painful to continue. The ages of the patients were from 17-70 years, with an average of 42 years. There were 15 males and 33 females. The length of history averaged 21 years in the males, 18 years in the females, with a mean of 19 years. The majority of the 
patients had had at least 5 years' treatment with drugs before they came to the trial. The patients were first seen by one of us (KJZ), who excluded structural lesions and other underlying causes. He then placed the patient in one of three categories, namely migraine-31, muscle tension headaches -7 , or a combination of these two-10. It should be emphasised that classification of a patient's headaches into one of these groups was not an important issue in this trial, and indeed it is clear that precise delineation of types of headache may not be feasible at present. ${ }^{3}$

There is a great variety in the severity and frequency of the headaches in any one patient and between different patients, and numerous intercurrent factors in the patient's life certainly contribute to the symptoms, and so a very stringent trial does not seem to be possible.

The patients were given two psychological tests on their first visit before treatment: Beck's Depression Inventory ${ }^{4}$ and Eysenck's Personality Inventory. ${ }^{5}$ The results of these tests were analysed at the end of the trial. On the Beck Inventory, those scoring 11 or more ${ }^{67}$ were considered separately, being classified as having depressive features; there were 10 patients in this category.

The 38 patients not showing depressive features were assessed on the Eysenck Inventory. With regard to neuroticism, of these 38 patients, 30 came within the limits of normal, and eight showed a higher neuroticism score. However, the mean neuroticism score of the sample was 10 (standard deviation 3.9) compared to the neuroticism score of the normal population of 9 (standard deviation 4.8). Thus these patients are no different from the normal population in this respect.

All the patients in the trial were randomly allocated either to medical treatment or to acupuncture. Of the 48 patients, 25 started with medical treatment and 23 with acupuncture. After 3 months, each patient was assessed by one of us (GDS) and was then asked to switch to the other kind of treatment; they were then reassessed after 3 further months' treatment. In the event, 18 who started with medical treatment switched to acupuncture and 11 starting with acupuncture switched to medical treatment. Thus only 29 had both forms of treatment.

With regard to prophylaxis, it will be realised that patients who had been having migraine or other headaches for about twenty years had tried every kind of drug that had been fashionable at that time. The main drug they were given when they entered this trial was propranolol; other drugs used were clonidine, pizotifen, metaclopramide, Migraleve and Migril.

During the period of acupuncture the patients were asked to stop taking prophylactic medication, but when they had a headache, they were permitted to take any drugs that they favoured. They were also recommended to try hot and cold packs, ice massage, deep massage to the neck and shoulder muscles and pressure on acupuncture points, during the migraine or headaches.

Acupuncture was performed by two of us (LL and PWN). It consisted of inserting and twiddling the needles by hand; sometimes electrical stimulation of the needles was also used. We did not attempt to assess the value of different methods of acupuncture. In all patients, local points tender on pressure were treated; in some cases distant points were also treated. The points were classical Chinese acupuncture points. ${ }^{8}$ Local points almost always included points just medial to the mastoid process (GB20), over the middle of upper border of the trapezius (GB21) and in the temporalis muscle. Distant points usually included the first dorsal interosseous muscle of the upper limbs ( $\mathrm{Li} \mathrm{4}$ ) and/or lower limbs (Liv 3). A minimum of six points was usually needled on each occasion. If a point was no longer tender after several treatments, it was no longer needled. Each local point was treated for about 2 min or until the pain of the needling subsided and the patient reported that the region felt numb.

The assessment of the prophylactic treatment was based on the patients' reports of their headaches. The patients kept a daily chart showing the number of headaches of each kind. They were questioned about the severity and duration of the headaches, their susceptibility to alleviating drugs, and about any changes in their lives related to changes in headaches. Although more complicated ways of assessing improvements were first considered, the patients themselves tended to assess the treatment as no benefit, slight benefit, moderate benefit and great improvement; we therefore adopted this simple classification.

\section{Results}

The results are shown in tables $1 \mathrm{a}, 1 \mathrm{~b}, 2$ and 3 . As

Table 1a Results of prophylaxis in all patients

\begin{tabular}{llllll}
\hline Kind of prophylaxis & \multicolumn{4}{l}{ Number of patients } \\
\cline { 2 - 6 } & Total & Great improvement & Moderate benefit & Slight benefit & No benefit \\
\hline Acupuncture & 41 & 9 & 7 & 8 & 17 \\
Medical & 36 & 3 & 1 & 5 & 27 \\
\hline
\end{tabular}

Table 1b Results of prophylaxis in 10 patients with depressive symptomatology

\begin{tabular}{llllll}
\hline Kind of prophylaxis & \multicolumn{4}{l}{ Number of patients } & \\
\cline { 2 - 6 } & Total & Great improvement & Moderate benefit & Slight benefit & No benefit \\
\hline Acupuncture & 7 & 3 & 1 & 1 & 2 \\
Medical & 7 & 1 & 0 & 1 & 4 \\
\hline
\end{tabular}


Table 2 Patients' assessment of treatment by acupuncture and medical treatment

\begin{tabular}{|c|c|c|c|c|c|c|}
\hline Course & & $\begin{array}{l}\text { Total } \\
\text { patients }\end{array}$ & $\begin{array}{l}\text { Great } \\
\text { improvement }\end{array}$ & $\begin{array}{l}\text { Moderate } \\
\text { improvement }\end{array}$ & $\begin{array}{l}\text { Slight } \\
\text { improvement }\end{array}$ & $\begin{array}{l}\text { No } \\
\text { benefit }\end{array}$ \\
\hline \multirow{4}{*}{ Acupuncture } & $\begin{array}{l}\text { As first treatment } \\
\text { (a) not changing to medical treatment }\end{array}$ & 12 & 6 & 2 & 2 & 2 \\
\hline & (b) changing to medical treatment & 11 & 0 & 0 & 1 & 10 \\
\hline & As second treatment & 18 & 3 & 5 & 5 & 5 \\
\hline & Total & 41 & 9 & 7 & 8 & 17 \\
\hline \multirow{4}{*}{ Medical Treatment } & $\begin{array}{l}\text { As first treatment } \\
\text { (a) not changing to acupuncture }\end{array}$ & 7 & 3 & 1 & 1 & 2 \\
\hline & (b) changing to acupuncture & 18 & 0 & 0 & 1 & 17 \\
\hline & As second treatment & 11 & 0 & 0 & 3 & 8 \\
\hline & Total & 36 & 3 & 1 & 5 & 27 \\
\hline
\end{tabular}

some patients did not change to the other form of prophylaxis, table 1a gives the outcome of the 41 courses of acupuncture and the 36 courses of medical prophylaxis. It will be seen that $24 / 41$ patients were better on acupuncture and 17 were not better. The medical treatment that the patients received here helped only a quarter of the patients $(9 / 36)$. Just under a fifth of the patients having acupuncture reported great improvement whereas a twelfth of those on medical treatment had great improvement. All the patients reporting great improvement, had a reduction in the frequency and severity of headaches. Of those having acupuncture with moderate improvement, five had a reduction in both, one in the frequency and one in the severity of headaches. Of those having slight benefit, two patients reported improvement in both, two a reduction in frequency and four a reduction in severity. Of those having medical treatment, the one showing moderate improvement had a reduction in both, and of the five with slight improvement, three had a reduction in both frequency and severity, and one each in severity and frequency.

Of the 10 patients showing depressive symptomatology presented in table $1 \mathrm{~b}$, five out of seven had improvement with acupuncture and none of these was helped by medical treatment for migraine and headaches. Two out of seven patients who had medical treatment had slight benefit; one had great improvement.
Table 2 further analyses the data given in table 1 paying attention to which treatment was given initially. Of the 23 patients having acupuncture as the first treatment, 12 refused to change to medical treatment; of the 25 who started with medical treatment, seven refused to change. It is clear from this table, that those who received much benefit from a treatment were unwilling to change and also that those who received medical treatment as their second treatment in this trial obtained minimal benefit.

The 29 patients who agreed to switch from one kind of treatment to the other did not receive great benefit from the first form of treatment they received. Their preferences are shown in table 3 . The patients' ratings of the treatments are given as 3 for great benefit, 2 for moderate, 1 for slight and 0 for no benefit. A patient's preference for one kind of treatment is indicated by subtracting a lower rating number from a higher number. For example, a patient rating acupuncture as 2 and medical treatment as 1 is entered as a preference for acupuncture of $2-1=1$ grade in the table. No preference is shown as 0 grade: this figure includes those helped by both forms of treatment and those helped by neither. The table shows that of these patients who had had both treatments, a larger proportion preferred acupuncture.

There are benefits of treatment by acupuncture not shown in these tables. Most patients were able to

Table 3 Comparison of patients' assessment of treatment by acupuncture and medical treatment

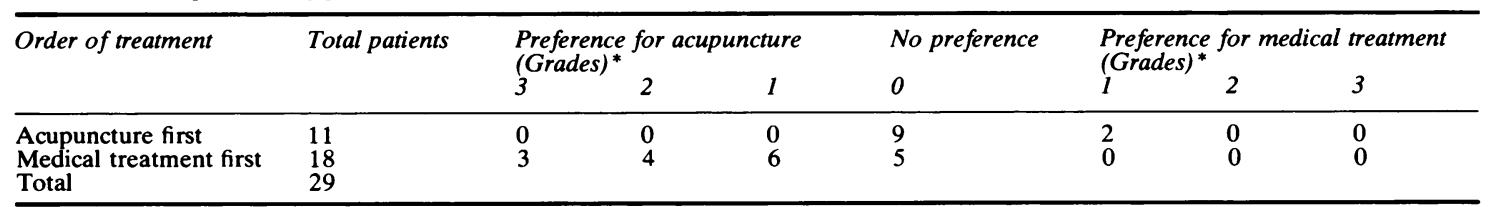

${ }^{*}$ For explanation of grading see text. 
reduce the dose of medication and others found that their headaches or migraine became responsive to analgesic drugs whereas they had not been so before. Further, all the patients having benefit from acupuncture gave up taking prophylactic medication to prevent headaches and migraine.

Acupuncture was effective in both muscle tension headaches and migraine. It might improve one sort of headache and not the other. Sometimes, when it improved the migraine, the muscle tension headaches would become more frequent. Many of the patients with muscle tension headaches said that they realised that before having acupuncture they felt "headachey" all the time, and that acupuncture had removed that symptom. Two patients found that acupuncture made their headaches worse, and three tended to feel faint during the treatment. Four patients felt drowsy and two felt euphoric. The patients who preferred drugs disliked the idea of having needles, and if they had acupuncture, strongly disliked the pain of it. The patients who preferred treatment with acupuncture were delighted to be able to stop taking medication, except during the time of a migraine attack or a headache exacerbation.

Whether the presence of tender points in the head and neck is related to the outcome of acupuncture treatment was examined; the relation between these two factors is shown in table 4 . It will be seen that $34 / 41$ patients had tender points. Treatment by acupuncture was likely to be successful when these points were present and to fail when they were absent.

A patient who was greatly improved by acupuncture is presented as an illustration. Mr AWW aged 61 years first attended hospital in 1968 at the age of 36 reporting that he had had migraine for the previous 8 years. Both his mother and his sister had migraine. At that time, he was having about one attack a month. The attacks would start with tingling in his hands and difficulty in focusing. He usually had a stiff tense feeling in the back of his neck. This would spread so that he had severe headache at the back of his head and in his forehead which would become "a terrible pain, feeling as though my head would burst." At the same time he saw flashing lights, usually more on the right than left. The headache then became a painful throbbing, synchronous with his pulse and made much worse by any movement. Nausea came on early in the attack; if it went on to vomiting, the attack usually finished. In addition, he

Table 4 Relation between presence of tender points and results of acupuncture treatment

\begin{tabular}{lllr}
\hline $\begin{array}{l}\text { Tender } \\
\text { points }\end{array}$ & $\begin{array}{l}\text { Acupuncture } \\
\text { beneficial }\end{array}$ & $\begin{array}{l}\text { Acupuncture } \\
\text { failed }\end{array}$ & Total \\
\hline Present & 23 & 11 & 34 \\
Absent & 1 & 6 & 7 \\
Total & 24 & 17 & 41 \\
\hline
\end{tabular}

had "ordinary headaches" on most of the days when he did not have migraine. At no time were there any abnormal neurological signs. His attacks increased steadily, in spite of (or probably because of) his taking $16 \mathrm{mg}$ of ergotamine daily; he was eventually weaned off this drug completely. He had been tested for food allergy, using the RAST method, and was found to be sensitive to milk, tea, cheese, egg, tomato, orange, fish, wheat, rice, grape and apple. The doctor who did this test recommended that the patient should avoid these foods but the patient did not do so.

When we first saw him 3 years ago, he reported that he was having three migraine attacks a week. He then entered this trial, starting treatment with acupuncture. Points GB 20 were tender bilaterally and were needled. Needling GB 20 on the right induced a slight sensation of migraine, with dryness of the mouth, nausea and flashing lights in the right visual field. On returning home 100 miles away by train, he vomited as he usually did in a migraine attack. One week later, he returned and reported that he had had four migraines, which was his usual number in a week at that time; and on the days which he had not had migraine, he had had "ordinary" headaches. However, the migraines had changed in character. Previously the pain had been in the forehead and in the neck; now it was only in the forehead. Local tender points in the forehead and temple (GB 3 and 14) were needled bilaterally. This again gave him migraine-like pain in the right forehead, nausea and sweating and made him feel unsteady and dizzy, which continued for the rest of the day. When he returned 2 weeks later, he reported he had had two migraines and five "ordinary" headaches; he had not had so many days free from headaches for 20 years; his neck stiffness had also gone. He had two more acupuncture treatments. Since the last treatment two years ago, he has had no further attacks of migraine although he may have an "ordinary" headache about once a week; and he has also felt more relaxed. For the first time for twenty years, he has been on no medication. He continues to take a normal diet and remains free from migraine and with a greatly reduced number of muscle tension headaches.

\section{Discussion}

It should be pointed out that the patients with headache and migraine who come to a hospital such as this differ from a cross-section of the population with these disorders. The patients who attend this hospital have mostly been to their general practitioners for many years with these complaints, and they or their doctors are not satisfied with the drug treatment they have had. As patients had not been helped by drugs, they might not have been expected to get benefit from a further trial of drugs. And indeed, only $9 / 36(25 \%)$ benefited from prophylaxis with drugs and in only four patients was there marked improvement. By comparison 24/41 patients $(59 \%)$ benefited from acupuncture, 16 getting marked benefit. Thirteen out of 29 (45\%) patients who had both forms of prophylaxis obtained no benefit from either. 
One of the lessons learned from treating patients with acupuncture is that the length of time the patient has had headaches or migraine does not affect the result. One of the best results in this series was in a patient who had had depressive symptoms and migraine for 50 years.

We were also surprised by the good results with acupuncture in those with depressive features: $3 / 7$ had great improvement. Standard medical treatment for muscle tension headaches and migraine produced no improvement in this group of patients. This figure has altered our practice: whereas formerly we did not treat these depressed patients with acupuncture we are willing to do so now, after we have discussed the situation with the patient and if the patient wishes to try acupuncture.

Acupuncture can remove, at least temporarily, headaches of organic origin. The following patients, though not in the present series, had relief from headache-two with giant-cell arteritis, one with a glioma and one with a pituitary tumour. It is important to realise, then, that successful treatment with acupuncture does not imply that the patient does not have a serious underlying condition.

With regard to predicting whether acupuncture is likely to help a patient or not, we conclude that when there are no local tender points acupuncture is less likely to help. The presence of local tender points, however, does not ensure a satisfactory result; nor does the degree of tenderness on pressure have any predictive value.

Our technique of acupuncture is painful for the patient and time-consuming for the doctor. It involves spending 20 minutes with the patient on a number of occasions. We always try acupuncture for at least three occasions before giving it up as a failure. We would therefore not suggest that anyone who has minor headaches responding to mild analgesic drugs should be treated with acupuncture.

One of the disadvantages of acupuncture is that the majority of patients need to come back for more treatment at intervals of 2 to 6 months. This means that eventually the clinic becomes filled with old patients and so it is difficult to provide new patients with the weekly treatment that they need at first. From the patient's point of view, acupuncture is a very safe method of treatment but it requires repeated visits to the clinic. It is also a cheap form of treatment, particularly as the patients usually stop taking prophylactic medication, which they are always pleased to do. We conclude that acupuncture is a beneficial treatment for headache and migraine for some patients and that its more widespread use is justified.

We are grateful to Dr Ian Sutherland, Director, MRC Biostatistics Unit, Cambridge, who helped us with the design of this trial and with the presentation of the results. This work was initiated when Dr PW Nathan was a member of the external staff of the Medical Research Council.

\section{References}

1 Millman BS. Acupuncture: context and critique. Am Rev Med 1977;28:223-34.

${ }^{2}$ Editorial: Treatment of migraine. Lancet 1982;1: 1338-40.

${ }^{3}$ Editorial: Classification of headache. Lancet 1982; 2: 1318

${ }^{4}$ Beck AT, Ward CH, Mendelson M, Mock J, Erbaugh J. An inventory for measuring depression. Arch Gen Psychiat 1961;4:53-63.

${ }^{5}$ Eysenck HJ, Eysenck SBG. Manual of the Eysenck Personality Inventory. London: Univeristy of London Press, 1964.

${ }^{-}$Metcalfe M, Goldman E. Validation of an inventory for measuring depression. Br J Psychiat 1965;111:240-2.

' Salkind MR. Beck depression inventory in general practice. J Roy Coll Gen Pract 1969;18:267-71.

${ }^{8}$ Mann F. Atlas of Acupuncture. London: Heinemann Medical Books.

${ }^{9}$ Monro J, Brostoff J, Carini C, Zilkha KJ. Food allergy in migraine: study of dietary exclusion and RAST. Lancet 1980;2:1-4. 